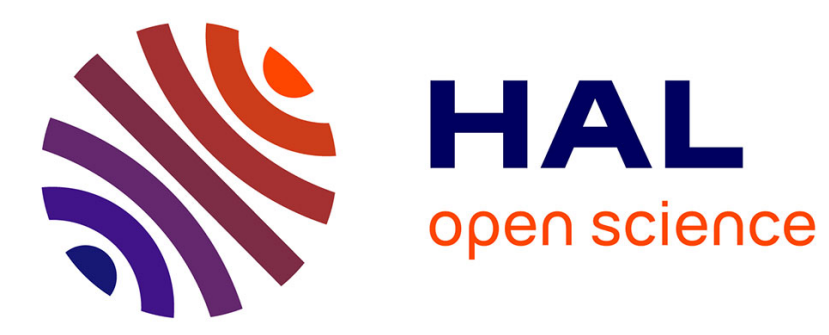

\title{
Unscented Kalman Observer
}

Assia Daid, Eric Busvelle, Mohamed Aidene

\section{To cite this version:}

Assia Daid, Eric Busvelle, Mohamed Aidene. Unscented Kalman Observer. 8th International Conference on Systems and Control, Oct 2019, Marrakesh, Morocco. hal-02286201

\section{HAL Id: hal-02286201 \\ https://hal.science/hal-02286201}

Submitted on 13 Sep 2019

HAL is a multi-disciplinary open access archive for the deposit and dissemination of scientific research documents, whether they are published or not. The documents may come from teaching and research institutions in France or abroad, or from public or private research centers.
L'archive ouverte pluridisciplinaire HAL, est destinée au dépôt et à la diffusion de documents scientifiques de niveau recherche, publiés ou non, émanant des établissements d'enseignement et de recherche français ou étrangers, des laboratoires publics ou privés. 


\title{
Unscented Kalman Observer*
}

\author{
Assia Daid ${ }^{1}$, Eric Busvelle ${ }^{2}$,and Mohamed Aidene ${ }^{3}$
}

\begin{abstract}
The extended Kalman filter is an exponentially converging observer as soon as it is written in a canonical form of observability and in its high-gain form. It is shown that unlike extended Kalman filter, unscented Kalman filter can not be an exponentially converging observer. We propose a slight modification of the unscented Kalman filter to build an exponentially converging observer called unscented Kalman observer. Performances of this new observer are illustrated on an example of geolocation problem.
\end{abstract}

\section{INTRODUCTION}

In this paper, we consider the observation problem of a nonlinear dynamic system in continuous time, which have the following form

$$
\left\{\begin{aligned}
\frac{d x(t)}{d t} & =f(x(t), t) \\
y(t) & =h(x(t), t)
\end{aligned}\right.
$$

$x(t) \in R^{n}, y(t) \in R^{m}$. The extended Kalman filter (EKF) is an important and widely used tool for the state estimation in such nonlinear dynamic systems (see [4], [12] in a deterministic setting and [18], [19] in the stochastic case, or [13] for a Matlab implementation including a complete discussion concerning EKF and its variants).

Generally, we distinguish two approaches for nonlinear state estimation. The first one is nonlinear filtering, which consists in studying the probability law of the state of a stochastic dynamic system conditionally to the observations. This is the usual setting for using EKF. The second approach is purely deterministic, it consists of constructing another dynamic system (the observer) that uses as input the available measurements and whose state converges asymptotically (and generally exponentially).

The extended Kalman filter is based on a linearization of the system along the estimated trajectory, the actual one being unknown, and using the equations of the linear Kalman filter. Although this biased linearization of the system prevents from analytically proving the observer's convergence for any initial error, such proofs exist when initial estimation errors are small enough (see [2], [7], [20], [21], [22]).

\footnotetext{
*This work was partially supported by PHC Tassili project, Campus France

${ }^{1}$ Assia Daid is with Laboratoire de Conception et Conduite des Systèmes de Production,and with Laboratoire d'Informatique et des Systèmes, UMR 7020 Université Mouloud MAMMERI,Tizi-Ouzou,Algérie, Université de Toulon, France assia.daid@univ-tln.fr

${ }^{2}$ Eric Busvelle is with Laboratoire d'Informatique et des Systèmes, UMR 7020, Université de Toulon, France eric.busvelle@univ-tln.fr

Mohamed Aidene is with Laboratoire de Conception et Conduite des Systèmes de Production, Université Mouloud MAMMERI,TiziOuzou,Algérie aidene@ummto.dz
}

However, first-order linearization can introduce significant errors when estimating the mean and covariance of the state. In addition, the derivation of Jacobian matrices is not easy in many applications (see, for instance, [16] and a way to work around the problem). This means that the EKF function is difficult to implement, to tune and its reliability is limited.

An other approach has been developed (introduced by Julier and Uhlmann in 1995, [15]) to improve the noise propagation through non-linearities of the system, called unscented Kalman filter (UKF). In fact, the only difference between EKF and UKF is how each filter handles mean and covariance propagation through the dynamic of the system. The UKF algorithm is based on the unscented transformation (UT) which is used for forming a Gaussian approximation of the joint distribution of gaussian random variables [23]. This transformation is briefly explain Section II-A.

Compared to extended Kalman filter, few works have been done to analyze the convergence of unscented Kalman filter. Some proof are established for nonlinear stochastic discretetime systems (see [25], [17], [10]) and in the stochastic continuous-time case (see [14]). Despite its interest (due to the fact that it does not require a priori Jacobian computation), UKF convergence has not being studied in a deterministic setting. Two reasons may explain this neglecting : the first one is that UKF is an improvement of EKF only because it tackles gaussian noise more efficiently, the second one because without modification, it is not convergent as we will see in Section II-B.

A successful approach to study the exponential convergence of the EKF is to use the high gain theory (see [12], [5]). It can be proved that, provided that the nonlinear system is put into a normal forms of observability (which is always possible if the system is observable in some sense, see [12]), and if gain is large enough, EKF converges for any initial error. With this structure, EKF is called kigh-gain extended Kalman filter (HG-EKF) and it is an exponential observer. The high-gain is computed by a modification of the covariance matrices $R$ and $Q$ (which are interpreted as cost matrices in deterministic case), by the use of a fixed scalar parameter (usually denoted $\theta$ ). The convergence is effective when $\theta$ is large enough (see for more explanations [4], [3], [12]).

Motivated by the use of the HG-EKF as a deterministic observer for nonlinear continuous time systems, and its good properties of convergence (both local and global), we study the convergence of an high-gain and non high-gain version of UKF for deterministic observer in continuous-time systems.

In Section II, we recall the unscented transformation, the unscented Kalman filter, and show that the unscented Kalman 
filter will not converge (in a deterministic setting) as soon as the system is nonlinear.

In Section III, we propose a modification which avoid the bias of UKF and we call our observer the unscented Kalman observer. We build the high-gain version of the UKO (called high-gain unscented Kalman observer, HG-UKO) and we give some convergence results. In fact, $\mathrm{HG}-\mathrm{UKO}$ is a local exponentially converging observer (see also [9]) and it becomes a global converging observer if we assume that the solution of the Riccati equation is bounded.

A simulation, very similar to the example presented in [11], has been done for the comparison of performance of each observers in Section IV.

\section{Continuous-time Unscented Kalman Filter}

In this section, we will briefly present the unscented Kalman filter in matrix form (see [23]). This filter uses $\sigma$-points (denoted by $X$ defined below) which capture the statistics of a Gaussian random variable. These $\sigma$-points prevent to compute the Jacobian matrices, which is very interesting, especially when non-linear functions are complicated functions (this is the case in the high-gain framework since these functions result from a non-linear change of coordinates, because of many Lie derivatives).

The UKF is based on the unscented transformation which is detailed in the following subsection.

\section{A. Unscented transformation}

The Unscented transformation is a non-linear method for calculating the statistics of a random variable, which undergoes a non linear transformation. The procedure to implement the UKF in continuous-time is summarized as follows [23], [24]

1) Choose $2 n+1 \sigma$-points:

$$
X=[m \cdots m]+\sqrt{c}\left[\begin{array}{lll}
0 & \sqrt{P} & -\sqrt{P}
\end{array}\right]
$$

$X$ is the matrix of $\sigma$-points, $c=\alpha^{2}(n+k)$, with $k \geq 0$, $\alpha \in(0,1] . c, k$ and $\alpha$ are setting parameters . Contrary to [23] where $\sqrt{P}$ is under defined (and arbitrarily chosen as the Cholesky factorization of $P$ ), in (2), the matrix square root $\sqrt{P}$ of the positive definite matrix $P$ is the unique positive definite matrix such that $P=\sqrt{P}^{2}$. This point is required for the proof of convergence.

2) Compute the associated weights of the $\sigma$-points, $w_{m}=\left(W_{m}^{0}, W_{m}^{1}, \ldots W_{m}^{2 n}\right)^{t}$ where

$$
\begin{aligned}
W_{m}^{(0)} & =\frac{\lambda}{n+\lambda}, \\
W_{m}^{(i)} & =\frac{1}{2(n+\lambda)}, \quad i=1, \ldots 2 n
\end{aligned}
$$

and $w_{c}=\left(W_{c}^{0}, W_{c}^{1}, \ldots W_{c}^{2 n}\right)^{t}$ where

$$
\begin{aligned}
& W_{c}^{(0)}=\frac{\lambda}{n+\lambda}+\left(1-\alpha^{2}+\beta\right), \\
& W_{c}^{(i)}=\frac{1}{2(n+\lambda)}, \quad i=1, \ldots 2 n
\end{aligned}
$$

$\lambda$ is a scalar parameter defined by $\lambda=c-n$.

3) Convert each $\sigma$-points by the nonlinear transformation

$g$

$$
Y=g(X)=\left[g\left(X_{0}\right) \cdots g\left(X_{2 n}\right)\right]
$$

where $X \in R^{n \times 2 n+1}, X_{i}$ denote the $i^{\text {th }}$ column of $X$, $g: R^{n} \rightarrow R^{m}$, and (3) means that the $i^{\text {th }}$ column $Y_{i}$ of the matrix $Y \in R^{m \times 2 n+1}$ is formed as follows:

$$
Y_{i}=g\left(X_{i}\right)
$$

4) The mean $\mathrm{E}[\mathrm{g}(\mathrm{X})]$ and covariance $\operatorname{Cov}(\mathrm{g}(\mathrm{X}))$ of $g(X)$ are estimated respectively by

$$
\mu_{u}=g(X) w_{m}=\sum_{i=0}^{2 n} W_{m}^{(i)} g\left(X_{i}\right)
$$

and

$$
S_{u}=\sum_{i=0}^{2 n} W_{c}^{(i)}\left(g\left(X_{i}\right)-m\right)\left(g\left(X_{i}\right)-m\right)^{t}
$$

5) The cross-covariance of $X$ and $g(X)$ is estimated as

$$
C_{u}=\sum_{i=0}^{2 n} W_{c}^{(i)}\left(X_{i}-m\right)\left(g\left(X_{i}\right)-\mu_{u}\right)^{t}
$$

The matrix $W$ is defined as

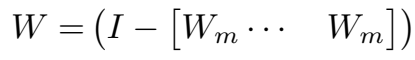

$$
\begin{aligned}
& \times \operatorname{diag}\left(\begin{array}{lll}
W_{c}^{0} & \cdots & W_{c}^{2 n}
\end{array}\right)
\end{aligned}
$$

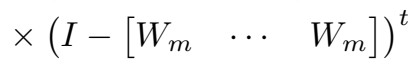

\section{B. Algorithm of UKF in continuous-time}

The equations corresponding to the UKF in the continuous-time are given by (see [23])

$$
\left\{\begin{aligned}
K(t) & =X(t) W h(X(t), t)^{t} R^{-1}(t) \\
\frac{d m(t)}{d t} & =f(X(t), t) w_{m}+K(t)\left(y(t)-h(X(t), t) w_{m}\right) \\
\frac{d P(t)}{d t} & =X(t) W f(X(t), t)^{t}+f(X(t), t) W X(t)^{t} \\
& +Q(t)-K(t) R(t) K(t)^{t}
\end{aligned}\right.
$$

In this algorithm $Q(t)$ and $R(t)$ represent covariance matrix of the state noise and covariance matrix of measurement noise respectively. They are both symmetric positive definite. In the deterministic case, these two matrices will be considered as tuning parameters (and usually independent from $t$ ). See [23], [24] for the transition from the unscented transformation to the UKF equations.

The unscented Kalman filter is not a good candidate as an exponential observer. Indeed, assuming that the function $h$ is linear, and suppose $x(t)=0$ is an equilibrium state. If $m(t)=0$, one expect that the observer remains at equilibrium state 0 (since the innovation term $y(t)-h(X(t), t) w_{m}$ is equal to zero). 
The state equation in (6) becomes

$$
\begin{aligned}
\frac{d m(t)}{d t} & =f(X(t), t) w_{m} \\
& =\frac{1}{2(n+\lambda)} \sum_{i=1}^{n} f\left((\sqrt{c P})_{i}\right)+f\left((-\sqrt{c P})_{i}\right)
\end{aligned}
$$

where $(\sqrt{c P})_{i}$ is the $i^{\text {th }}$ column of $\sqrt{c P}$. The right term of (7) is equal to zero if $f$ is linear or only odd but if not, it is different from zero and therefore, 0 is not an equilibrium state of (6).

\section{High-GAIN UNSCENTED KALMAN OBSERVER}

\section{A. Unscented Kalman Observer}

The solution of the previous problem is quite obvious. Indeed, using the state equation that describes the evolution of EKF in (6), we obtain the following new equation describing our unscented Kalman observer (UKO) :

$$
\left\{\begin{aligned}
K(t) & =X(t) W h^{t}(X(t), t) \\
\frac{d m(t)}{d t} & =f(m(t), t)+K(t)[y(t)-h(m(t), t)] \\
\frac{d P(t)}{d t} & =X(t) W f^{t}(X(t), t)+f(X(t), t) W X^{t}(t) \\
& +Q(t)-K(t) R(t) K^{t}(t)
\end{aligned}\right.
$$

In the stochastic case, this filter add nothing more to EKF (unlike UKF), but when used as an observer, UKO allows to construct an unbiased high-gain observer (HG-UKO) that converges exponentially. We will now study this observer as well as its high-gain version.

\section{B. High-gain extended Kalman filter}

The high-gain observer presented in this section is similar to the one developed in [12]. It is shown in this book that if a system has the property of being observable for all inputs, then there is a coordinate transformation such that a multi-input single-output system can be written in canonical form of observability. The high-gain observer consists to write the observer in these new coordinates and to redefine the matrices $Q$ and $R$ so that they depend on a parameter that is supposed to be "high enough" (the property "high enough" being explicited in [12]). Although less canonical, this method can be apply to multi-input multi-output systems. From now, we suppose that our system (1) is written in the new coordinates:

$$
\left\{\begin{array}{l}
\dot{x}(t)=A x(t)+b(x(t), t) \\
y(t)=C x(t)
\end{array}\right.
$$

where $x(t) \in R^{n}$ and $y(t) \in R$ are the state and the measured outputs respectively. For simplicity, we assumed that the dimension of the output is 1, to facilitates the definition of the normal form. For the general case, see for example [3]. The matrices $A, C$ are defined as follows:

$$
A=\left(\begin{array}{ccccc}
0 & 1 & 0 & \cdots & 0 \\
0 & 0 & 1 & \ddots & \vdots \\
0 & 0 & 0 & \ddots & 0 \\
\vdots & & \ddots & & 1 \\
0 & & \cdots & & 0
\end{array}\right) \text { and } C=\left(\begin{array}{lll}
1 & 0 & \cdots
\end{array}\right)
$$

Vector $b(x(t), t)$ is assumed to be triangular and compactly supported as follows :

$$
b(x(t), t)=\left(\begin{array}{c}
b_{1}\left(x_{1}(t), t\right) \\
b_{2}\left(x_{1}(t), x_{2}(t), t\right) \\
\vdots \\
b_{n}(x(t), t)
\end{array}\right)
$$

$L_{b}$ is the bound of the Jacobian matrix $b^{*}(x(t), t)$ of $b(x(t), t)$ with respect to $x$, that is to say $\left\|b^{*}(x(t), t)\right\| \leq L_{b}$.

Function $b(x(t), t)$ is supposed to be uniformly Lipschitz in $x$ independently of $t$ :

$$
\left\|b\left(x_{1}, t\right)-b\left(x_{2}, t\right)\right\| \leq L_{b}\left\|x_{1}(t)-x_{2}(t)\right\|
$$

It is well known that system (9) admits the following observer (called high-gain extended Kalman filter, HG-EKF:

$$
\left\{\begin{aligned}
\frac{d m}{d t} & =A m+b(m, t)+P C^{t} R^{-1}(y(t)-C m) \\
\frac{d P}{d t} & =\left(A+b^{*}(m, t)\right) P+P\left(A+b^{*}(m, t)\right)^{t} \\
& +Q^{\theta}-P C^{t} R^{-1} C P
\end{aligned}\right.
$$

where $i^{\text {th }}$ line and $j^{\text {th }}$ column of $Q^{\theta}$ are equal to $Q_{i, j}^{\theta}=$ $\theta^{i+j+1} Q_{i, j}$. This observer is described, among others, in [4]. This is a classical EKF, but written in canonical coordinates and with a particular $Q$ matrix $Q^{\theta}$.

\section{High-gain unscented Kalman observer in final form}

The equations corresponding to HG-UKO in the continuous-time for the system (9) are given by

$$
\left\{\begin{aligned}
\frac{d m}{d t}= & A m+b(m, t)+P(t) C^{t} R^{-1}(y(t)-C m) \\
\frac{d P}{d t}= & X W\left(A X+b(X, t)^{t}+(A X+b(X)) W X^{t}\right. \\
& +Q^{\theta}-X W X^{t} C^{t} R^{-1} C X W X^{t}
\end{aligned}\right.
$$

where $X$ is the same matrix as defined in (2).

The matrix $Q^{\theta}$ is defined as in (10). These equations are obtained just as [23], [24], but using the equation of unscented Kalman Observer (UKO) in its high-gain version.

\section{Main results}

Theorem 1: Let us consider the Riccati equation of the UKO (8), and let us suppose that $\mathrm{Q}(\mathrm{t})$ and $\mathrm{R}(\mathrm{t})$ are bounded symmetric definite matrix (with lower bound greater than 0 ), $\mathrm{f}$ and $\mathrm{h}$ are $C^{2}$ functions with bounded second derivatives, assuming that the system is completely uniformly observable (standard hypothesis, see [1]), then for any $T>0$, there 
exists $c$ small enough such that for all $t>T, P(t)$ (as well as $S(t)=P^{-1}(t)$ ) remains bounded from below (by a strictly positive number) and from above.

Proof: Bounds on the solution of the Riccati equation in the Kalman filtering context are classical. In the following, we will use the approach developed in [12]. This proof is rather long but simple. It applies to the following Riccati equation written in $S(t)$ :

$$
\begin{array}{r}
\frac{d S(t)}{d t}=-A(t)^{t} S(t)-S(t) A(t)+C(t)^{t} R(t)^{-1} C(t) \\
-S(t)(Q(t)+\Omega(t)) S(t)
\end{array}
$$

where $A(t)$ and $C(t)$ are Jacobian matrix of $f$ and $h$ respectively, and where $R(t), Q(t)$ and $\Omega(t)$ are bounded symmetric matrix, $Q(t)+\Omega(t)$ and $R(t)$ being symmetric definite positive matrix. More precisely, we assume that there exists $\underline{r}, \bar{r}, q, \bar{q}$, and $\omega$ such that

- $0<\underline{r} I d \leq R(t) \leq \bar{r} I d$

- $0<\underline{q} I d \leq Q(t) \leq \bar{q} I d$

- $-\omega \overline{I d} \leq \Omega(t) \leq \omega I d$ with $\omega<\underline{q}$ such that $Q(t)+\Omega(t)$ is symmetric definite positive.

Under these conditions, using a slight modification of the proof in [12], there exists $0<\alpha<\beta$ such that

$$
\alpha I d \leq S(t) \leq \beta I d
$$

(in [12], $Q$ and $R$ are supposed to be constant matrix but it is not a big deal to generalize to time-dependant bounded matrix). The role of $\Omega(t)$ will be explain later.

Let us rewrite the Riccati equation in (8) in $S(t)$ rather than $P(t)$ (using $\frac{d}{d t}(P(t) S(t))=0$ ), it becomes after Taylor series expansion up to order 2 :

$$
\begin{aligned}
& \frac{d S}{d t}=- A^{t} S-S A+C^{t} R^{-1} C-S Q S \\
&+\frac{\sqrt{c}}{4} \sqrt{S} \mathcal{H}_{h}^{t} R^{-1} C+\frac{\sqrt{c}}{4} C^{t} R^{-1} \mathcal{H}_{h} \sqrt{S} \\
& \quad-\frac{\sqrt{c}}{4} \sqrt{S} \mathcal{H}_{f}^{t} S-\frac{\sqrt{c}}{4} S \mathcal{H}_{f}^{t} \sqrt{S} \\
&-\frac{c}{16} \sqrt{S} \mathcal{H}_{h}^{t} R^{-1} \mathcal{H}_{h} \sqrt{S}
\end{aligned}
$$

where $\mathcal{H}_{f}$ and $\mathcal{H}_{h}$ are large matrix involving second derivatives of $f$ and $h$ respectively, at some points depending from the position of $\sigma$-points. Let us rewrite (13) more simply

$$
\frac{d S}{d t}=-A^{t} S-S A+C^{t} R^{-1} C-S(Q+\Omega) S
$$

where $S(t) \Omega(t) S(t)$ are the five last terms in (13). A key point is that

$$
\|\Omega(t)\| \leq \sqrt{c} K(f, g, \underline{r}, \bar{r}, \underline{q}, \bar{q}, \min (S), \max (S))
$$

where $K$ is a bound depending from several parameters, including lower and upper bounds on $S(\min (S)$ and $\max (S)$ are scalar bounds in the sense of symmetric matrices partial ordering). Aside its dependence from bounds on $S$, the bound $K$ is a consequence of the hypothesis in the theorem ( $f$ and $h$ have bounded second derivatives) and the existence of bounds on $R$ and $Q$.
To prove that $S(t)$ remains bounded in the Riccati equation (13), we use an ad absurdum argument. Given $\omega>0$, let $\alpha$ and $\beta$ given by (12). Let $c$ small enough such that

$$
\sqrt{c} K\left(f, g, \underline{r}, \bar{r}, \underline{q}, \bar{q}, \frac{\alpha}{2}, 2 \beta\right)<\omega
$$

Let $S(0)$ be a matrix such that $\alpha I d<S(0)<\beta I d$. So, let us suppose that there exists $t_{0}$ being the largest time such that

$$
\alpha I d \leq S(t) \leq \beta I d \quad \forall t \leq t_{0}
$$

which is our ad absurdum hypothesis. By continuity of $S(t)$, there exists $t_{1}>t_{0}$ such that $\frac{\alpha}{2} I d \leq S(t) \leq 2 \beta I d$ for all $t \leq t_{1}$. Therefore, for all $t \leq t_{1},\|\Omega(t)\| \leq \omega$ from (15) and (16). Hence, applying classical bounds on Riccati equations (12), for all $t \leq t_{1}, \alpha I d \leq S(t) \leq \beta I d$. This is a contradiction since $t_{1}>t_{0}$ and $t_{0}$ is supposed to be the largest time satisfying (17).

As in [6] and [12] for instance, the following theorem is a direct consequence of the previous one.

Theorem 2: Under hypotheses of Theorem 1, the unscented Kalman observer (8) is a locally exponentially converging observer.

The high-gain construction permits us to extend this result from local to global. The bounds on the solution of the Riccati equation in (11) remains the main bottleneck for a mathematical result. Following the classical approach (see [12]), we guess that HG-UKO will be an exponentially converging observer as soon as $\theta$ is large enough and $c$ small enough, under the same conditions as in Theorem 1. Actually, we have the following result:

Theorem 3: Let us suppose that for a given $T>0$, there exists two constants $m_{1}$ end $m_{2}$ such that for any value of $\theta, 0<m_{1} \mathrm{Id} \leq S(t) \leq m_{2} \mathrm{Id}$, then there exists $\theta$ and $c$ such that the high-gain unscented Kalman observer (11) is a globally exponentially converging observer for of the system (9).

In Theorem 3, $S(t)$ is supposed to be bounded independently from $\theta$ for $t>T$. This hypothesis may looks very restrictive. In fact, it is verified for HG-EKF, see [6], [12]).

Complete proofs of these theorems are very technical and rather long. They consist mainly in straightforward adaptations of the proof in the book [12] and some analytic computations (such as Taylor series expansion to obtain (13) which use also some identities from the unscented transformation, see [23]). Theorems 1 and 2 are proved in details in [9]. Theorem 3 is a very simple result and it is proved in the Ph.D. of Assia Daid, in preparation. But Theorem 1 is clearly the "angular stone" of our new result.

\section{ILLUSTRATIVE EXAMPLE}

Let us consider a boat moving in the half-plane $x_{2}>$ 0 (the half-plane $x_{2}<0$ corresponds to the land), at an unknown speed in an orthonormal frame $\left(x_{1}, x_{2}\right)$. In order to estimate its position, two beacons are used ( denoted $A$ and $B$ ), and the boat continuously measures the directions of these beacons in relation to the north, given by an electronic compass. By using the angle between the position of the 
boat and each beacon, we want to estimate its instantaneous position.

The state of the system is denoted $x(t)=$ $\left(x_{1}(t), x_{2}(t), \alpha(t)\right), \quad \alpha$ being the angle between the direction of the boat and the north. The geolocation problem is expressed as the following Dubin's car dynamic model:

$$
\left\{\begin{aligned}
\dot{x_{1}}(t) & =u(t) \cos (\theta(t)) \\
\dot{x_{2}}(t) & =u(t) \sin (\theta(t)) \\
\dot{\alpha}(t) & =v(t)
\end{aligned}\right.
$$

Measurements are given by the following equations, assuming without loss of generality that the two beacons have respective positions $(0,0)$ for $A$ and $(1,0)$ for $B$.

$$
\left\{\begin{array}{l}
y_{1}(t)=\arctan \frac{x_{2}(t)}{x_{1}(t)} \\
y_{2}(t)=\arctan \frac{x_{2}(t)}{\left(x_{1}(t)-1\right)}
\end{array}\right.
$$

To apply the HG-UKO, we put (18) and (19) into a canonical form of observability, by using an appropriate change of variable as follows

$$
\phi(x(t))=\left(\begin{array}{c}
\arctan \frac{x_{2}(t)}{x_{1}(t)} \\
\arctan \frac{x_{2}(t)}{\left(x_{1}(t)-1\right)} \\
\frac{x_{1}(t) \sin \alpha(t)-x_{2}(t) \cos \alpha(t)}{x_{1}(t)^{2}+x_{2}(t)^{2}} \\
\frac{\left(x_{1}(t)-1\right) \sin \alpha(t)-x_{2}(t) \cos \alpha(t)}{\left(x_{1}(t)-1\right)^{2}+x_{2}(t)^{2}}
\end{array}\right)=\xi(t)
$$

In canonical coordinates the system (18) becomes

$$
\left(\begin{array}{c}
\dot{\xi}_{1}(t) \\
\dot{\xi_{2}}(t) \\
\dot{\xi_{3}}(t) \\
\dot{\xi_{4}}(t)
\end{array}\right)=\left(\begin{array}{c}
u \xi_{3}(t) \\
u \xi_{4}(t) \\
\left(v-2 u \xi_{3}(t)\right) \frac{x_{1}(t) \cos \alpha(t)+x_{2}(t) \sin \alpha(t)}{x_{1}(t)^{2}+x_{2}(t)^{2}} \\
\left(v-2 u \xi_{4}(t)\right) \frac{\left(x_{1}(t)-1\right) \cos \alpha(t)+x_{2}(t) \sin \alpha(t)}{\left(x_{1}(t)-1\right)^{2}+x_{2}(t)^{2}}
\end{array}\right)
$$

with measures $y_{1}(t)=\xi_{1}(t)$ and $y_{2}(t)=\xi_{2}(t)$.

As already said, even with this simple system, the change of coordinates involves quite complicated functions and we are quite happy to avoid Jacobian matrices computations.

\section{A. Comparative study}

For testing, we will assume that the boat follows a circular trajectory at a constant speed $V$, by taking the two constant controls $u(t)=V$ and $v(t)=\omega=\frac{V}{R}$, and by choosing $x_{1}(0)=c_{1}, x_{2}(0)=c_{2}-R, \alpha(0)=0$, the solution is

$$
\left\{\begin{aligned}
x_{1}(t) & =R \sin (\omega t)+c_{1} \\
x_{2}(t) & =-R \cos (\omega t)+c_{2} \\
\alpha(t) & =\omega t
\end{aligned}\right.
$$

and we have $\sqrt{\dot{x_{1}}(t)^{2}+\dot{x_{2}}(t)^{2}}=V$.

First, we compare UKO and HG-UKO. Both are used as observers, but the second is applied in the canonical form of observability and with a high-gain $\theta$. This high-gain is taken equal to 2 in all simulations.

The initial state of the system is $x_{0}=(3,1.5,0)$, and the initial state of the observer is $m=(3.5,3,1)$.

Estimated states from UKO and $\mathrm{HG}-\mathrm{UKO}$ are compared to actual state on Figure 1. Figure 2 represents the same
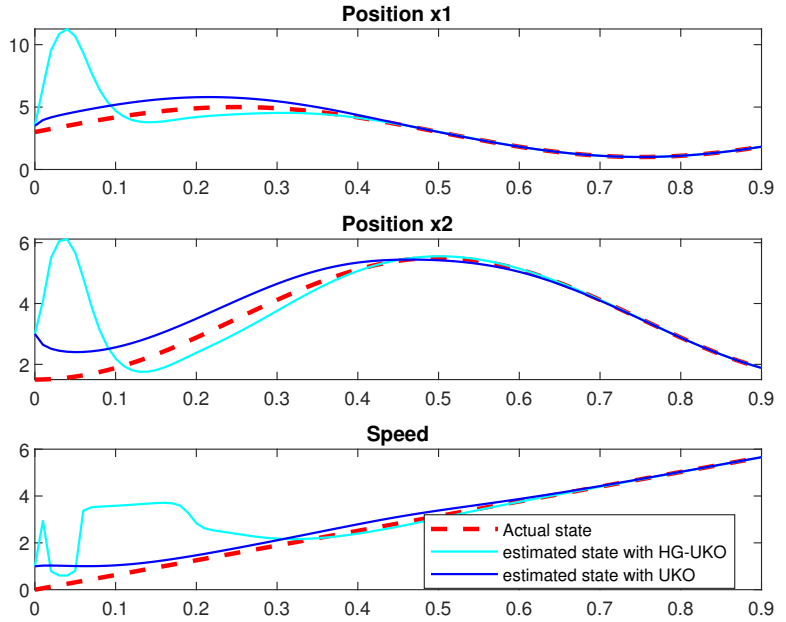

Fig. 1. Estimated states with UKO and HG-UKO

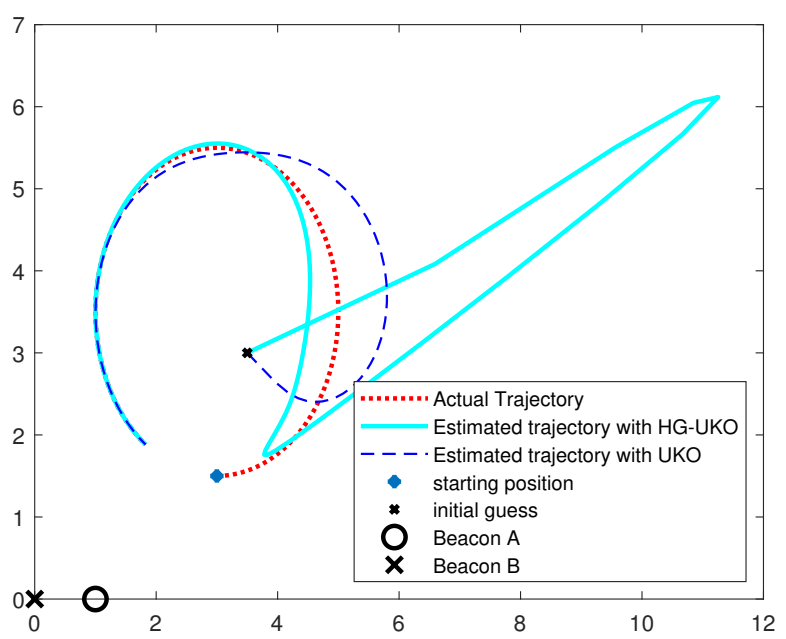

Fig. 2. Trajectory estimation with UKO and HG-UKO

simulation result in the state space. Not surprisingly, UKO and HG-UKO converge to the actual state, but the convergence of the HG-UKO is faster than UKO (in fact, it can be arbitrarily fast but in this case, it will be very sensitive to noise and we chose a value of $\theta$ which is a compromise between convergence and robustness).

We seen in Section II-B, that the HG-UKF can not converge as an observer. Figures 3 and 4 clearly shows the inevitable static error with HG-UKF, while HG-UKO has no bias. The error is particularly important and visible on Figure 4, as expected.

Next, we compared HG-UKO with HG-EKF, Figure 5, and Figure 6 shows that the two observers have quite similar performance : they converge at the same time with similar amplitude error. This is not a surprise since in this context, HG-UKO is more or less a numerical approximation of HG$\mathrm{EKF}$, in the sense that formal Jacobian matrix computation in HG-EHF is replaced by a kind of numerical approximation, based on difference quotients in HG-UKO.

It should be noted that we didn't put noise into these simulations, as we only test the performance of (HG-) UKO 

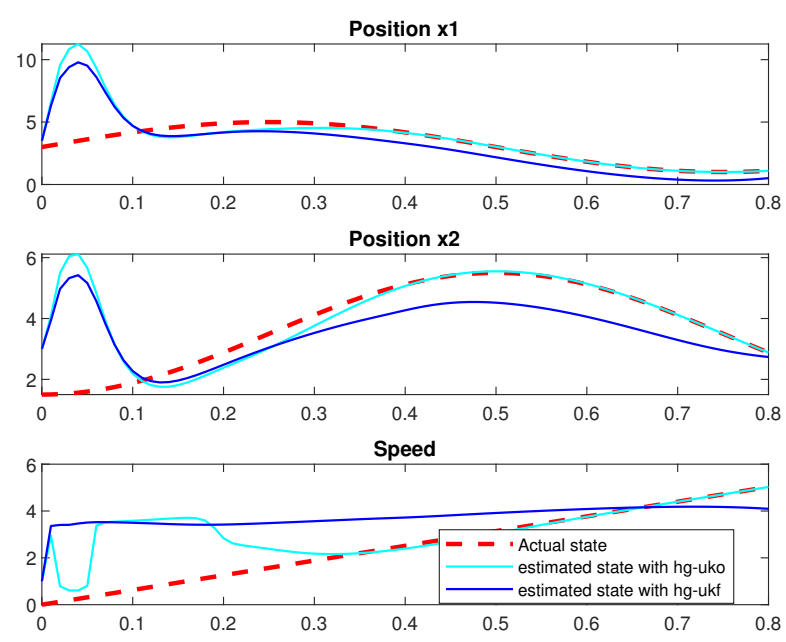

Fig. 3. Estimated states with HG-UKO and HG-UKF

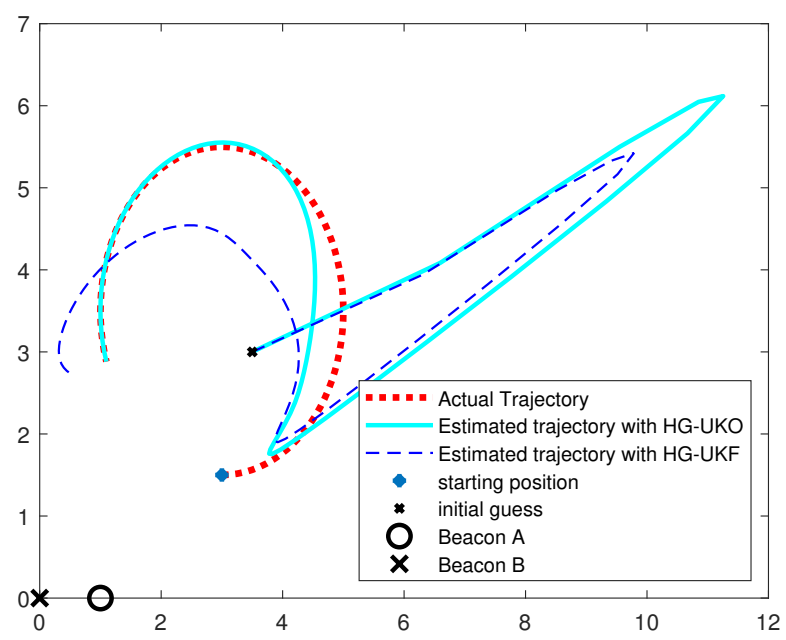

Fig. 4. Trajectory estimation with HG-UKO and HG-UKF
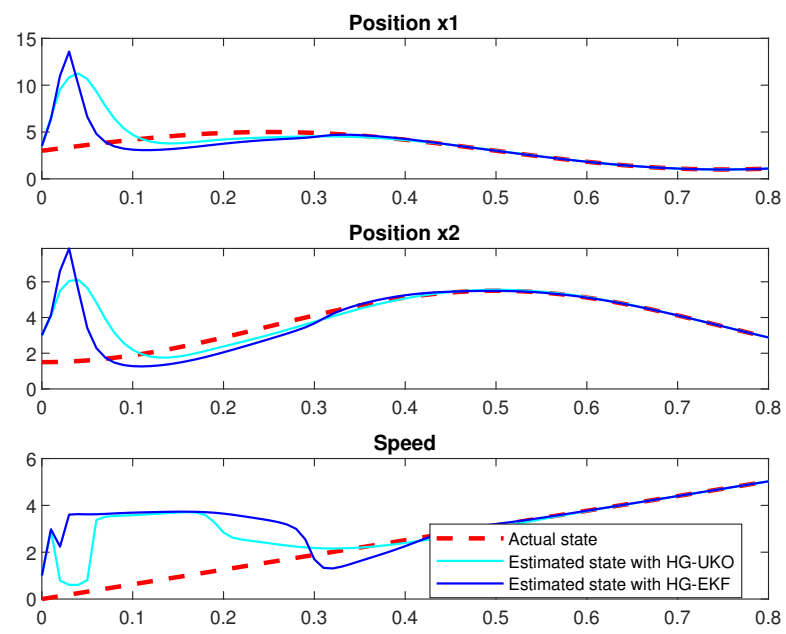

Fig. 5. Estimated states with HG-UKO and HG-EKF

as an observer. Nevertheless, in order to test the robustness of HG-UKO, we introduceunknown perturbations on outputs. More precisely, we add some trigonometric functions of the

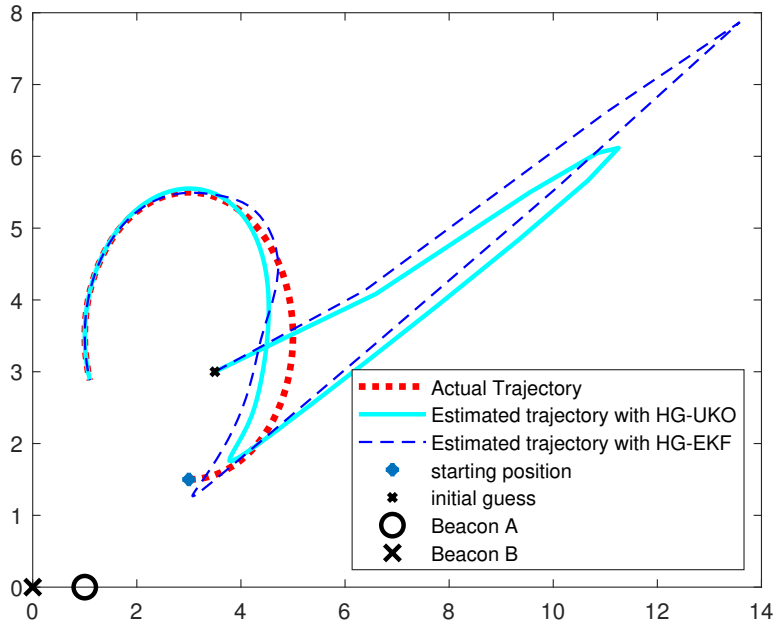

Fig. 6. Trajectory estimation with HG-UKO and HG-EKF
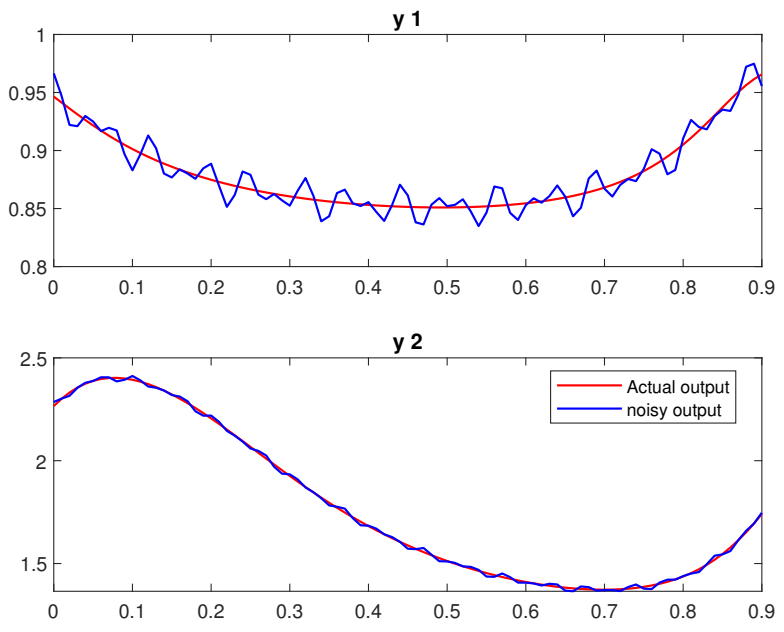

Fig. 7. Actual and noisy output

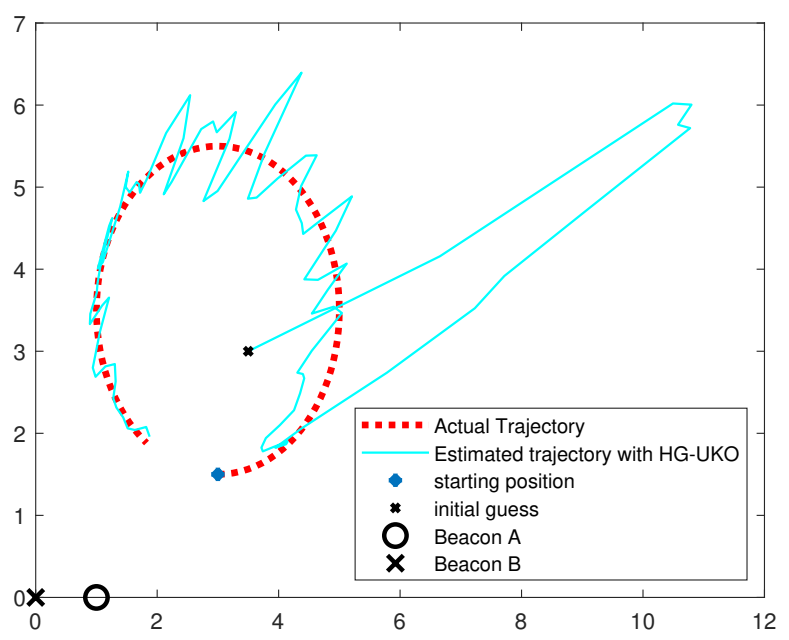

Fig. 8. Trajectory estimation with additive noise on the measurement

form $0.01\left(\cos \left(\omega_{i}^{(1)} t\right)+\cos \left(\omega_{i}^{(2)} t\right)\right)$ to each output $y_{i}, i=$ 1,2 corresponding to a perturbation with standard deviation equal to $1 \%$ for $y_{1}$ and $0.5 \%$ for $y_{2}$ (see Figure 7 ). The value 
of the high-gain parameter has not been adapted. Results are shown Figure 8. Although the noise induce some lack of performance, we see that convergence remains and is very satisfactory.

\section{CONCLUSION}

Several nonlinear observers were presented, among them only HG-EKF and HG-UKO are global nonlinear converging observers, while HG-UKF is not an observer (and has never been presented as is in litterature).

The advantage of HG-UKO is a relative simplicity of writing since it is not necessary to calculate any Jacobian matrices. This property may be particulary interesting when the system is written in its canonical form, since it is usually a rather complicated form involving high order Lie derivatives. These results have been established for continuous systems, and should be generalized to continuous-discrete systems.

\section{REFERENCES}

[1] G. Bornard, F. Celle-Couenne and G. Gilles, "Observability and observers", In: Fossard A.J., Normand-Cyrot D. (eds) Nonlinear Systems. Springer, Boston, MA, pp. 173-216, 1995.

[2] J. S. Baras, A. Bensoussan and M. R. James, "Dynamic observers as asymptotic limits of recursive filters: Special cases", SIAM Journal on Applied Mathematics, 48(5):1147-1158, 1988.

[3] N. Boizot "Adaptative high-gain extended Kalman filter and applications" , PhD thesis, Université de Bourgogne, Université du Luxembourg, 2010.

[4] N. Boizot and E. Busvelle, "Adaptive-gain observers and applications," In Nonlinear Observers and Applications, pages 71-114. Springer Berlin Heidelberg, 2007.

[5] N. Boizot, E. Busvelle and J.-P. Gauthier, "An adaptive high-gain observer for nonlinear systems", Automatica, 46(9):1483-1488, sept. 2010.

[6] N. Boizot and E. Busvelle, "On the stability of a differential Riccati equation for continuous-discrete observers", International Journal of Control, 89:2, pp. 322-336, 2016

[7] M. Boutayeb, H. Rafaralahy and M. Darouach, "Convergence analysis of the extended kalman filter used as an observer for nonlinear deterministic discrete-time systems", IEEE transactions on automatic control, 42(4):581-586, 1997.

[8] E. Busvelle and J.-P. Gauthier, "High-gain and non-high-gain observers for nonlinear systems", In Contemporary Trends in Nonlinear Geometric Control Theory and Its Applications, pp 257-286, Jan 2002.

[9] A. Daid, E. Busvelle, and M. Aidene, "On the convergence of the Unscented Kalman Filter", Submitted, 2019.

[10] G. Dymirkovsky, "New results on stochastic stability of discretetime unscented Kalman filter", In 7th IEEE Conference on Industrial Electronics and Applications (ICIEA), pages 1543-1548. IEEE, 2012.

[11] A. Feddaoui-Papin, N. Boizot, E. Busvelle, and V. Hugel, "Continuous-Discrete High-Gain Extended Kalman Filter for Mobile Robot with Asynchronous Outputs", Submitted to the 8th International Conference on Systems and Control (ICSC'19), Marrakesh, Morocco, October 23-25, 2019.

[12] J.-P. Gauthier and I. Kupka "Deterministic Observation Theory and Applications", Cambridge University Press, 2001.

[13] S. Hartikainen, A. Solin, and S. Särkkä, "Optimal filtering with kalman filters and smoothers", Department of Biomedica Engineering and Computational Sciences, Aalto University School of Science, 16th August, 2011.

[14] J. Xu, S. Wang, G. M. Dimirovski and Y. Jing, "Stochastic stability of the continuous-time unscented Kalman filter", In 47th IEEE Conference on Decision and Control, pages 5110-5115. IEEE, 2008.

[15] S. J. Julier, J. K. Uhlmann and H. F. Durrant-Whyte "A new approach for filtering nonlinear systems", In Proceedings of 1995 American Control Conference, 1995.

[16] F. Lafont, E. Busvelle and J.-P. Gauthier, "An adaptive high-gain observer for wastewater treatment systems", Journal of Process Control, 21(6):893-900, 2011.
[17] L. Li and Y. Xia, "Stochastic stability of the unscented Kalman filter with intermittent observations", Automatica, 48(5):978-981, may 2012.

[18] E. Pardoux, "Filtrage non linéaire et équations aux dérivées partielles stochastiques associées", In Ecole d'Eté de Probabilités de Saint-Flour XIX 1989, pages 68-163. Springer, 1991.

[19] J. Picard, "Efficiency of the extended Kalman filter for nonlinear systems with small noise", SIAM Journal on applied mathematics, 51(3):843-885, 1991.

[20] K. Reif, S. Gunther, E. Yaz and R. Unbehauen, "Stochastic stability of the discrete-time extended Kalman filter", IEEE Transactions on Automatic control, 44(4):714-728, 1999.

[21] K. Reif, F. Sonnemann and R. Unbehauen, "Modification of the extended Kalman filter with an additive term of instability", In Proceedings of 35th IEEE Conference on Decision and Control, volume 4, pages 4058-4059. IEEE, 1996.

[22] K. Reif, F. Sonnemann and R. Unbehauen, An EKF-based nonlinear observer with a prescribed degree of stability, Automatica, 34(9):11191123,1998

[23] S. Särkkä, "On unscented Kalman filtering for state estimation of continuous-time nonlinear systems", IEEE Transactions on Automatic Control, 52(9):1631-1641, sept. 2007.

[24] S. Särkkä, "Bayesian Filtering and Smoothing", Cambridge University Press, 2009.

[25] K. Xiong, H. Y. Zhang and C. W. Chan, "Performance evaluation of UKF-based nonlinear filtering", Automatica, 42(2):261-270, 2006. 